\title{
Testing and Diagnosis of Realistic Defects in Digital Microfluidic Biochips*
}

\author{
FEI SU \\ Intel Corporation, Folsom, CA, USA \\ fs@ee.duke.edu \\ WILLIAM HWANG \\ St John's College, University of Oxford, Oxford, UK \\ wlh@ee.duke.edu \\ ARINDAM MUKHERJEE \\ Department of Electrical and Computer Engineering, University of North Carolina at Charlotte, \\ Charlotte, NC 28223, USA \\ amukherj@uncc.edu \\ KRISHNENDU CHAKRABARTY \\ Department of Electrical and Computer Engineering, Duke University, Durham, NC 27708, USA \\ krish@ee.duke.edu
}

Received March 9, 2006; Revised July 8, 2006

Editor: M. Tehranipoor

\begin{abstract}
Microfluidics-based biochips are soon expected to revolutionize biosensing, clinical diagnostics and drug discovery. Robust off-line and on-line test techniques are required to ensure system dependability as these biochips are deployed for safety-critical applications. Due to the underlying mixed-technology and mixed-energy domains, biochips exhibit unique failure mechanisms and defects. We first relate some realistic defects to fault models and observable errors. We next set up an experiment to evaluate the manifestations of electrode-short faults. Motivated by the experimental results, we present a testing and diagnosis methodology to detect catastrophic faults and locate faulty regions. The proposed method is evaluated using a biochip performing real-life multiplexed bioassays.
\end{abstract}

Keywords: biosensors, biochips, microfluidics, defects, fault modeling, testing, diagnosis

\section{Introduction}

Over the past decade, research in integrated circuit testing has broadened from digital test to include the testing of analog and mixed-signal devices. More recently, new test techniques for mixed-technology microelectromechanical systems (MEMS) are also receiving attention $[2-4,9,10]$. As MEMS rapidly evolve from single components to highly integrated systems for safety-critical applications, dependability is emerging as an important performance parameter. Fabrication techniques such as silicon micro-

\footnotetext{
*This research was supported by the National Science Foundation under grants IIS-0312352 and CCF-0541055. A preliminary version of this paper appeared in Proc. IEEE International Test Conference, pp. 487-496, 2005.
}

machining lead to new types of manufacturing defects in MEMS [2]. Moreover, due to their underlying mixed technology and multiple energy domains (e.g., electric, mechanical, and fluidic), such composite microsystems exhibit failure mechanisms that are significantly different from those in electronic circuits. In fact, the 2003 International Technology Roadmap for Semiconductors (ITRS) recognizes the need for new test methods for disruptive device technologies that underly composite microsystems, and highlights it as one of the five difficult test challenges beyond 2009 [5].

Microfluidics-based biochips constitute an emerging category of mixed-technology microsystems [24]. Recent advances in microfluidics technology have led to the design and implementation of miniaturized devices for 
various biochemical applications. These microsystems, referred to interchangeably in the literature as microfluidics-based biochips, lab-on-a-chip and bioMEMS [12, 16], promise to revolutionize biosensing, clinical diagnostics and drug discovery. Such applications can benefit from the small size of biochips, the use of microliter/ nanoliter sample volumes, lower cost, and higher sensitivity compared to conventional laboratory methods.

The first generation of microfluidics-based biochips was based on the manipulation of continuous liquid flow through fabricated microchannels [24]. Liquid flow was achieved either by external pressure sources, integrated mechanical micropumps, or by electrokinetic mechanisms such as electro-osmosis. Recently, a novel microfluidics technology has been developed to manipulate liquids as discrete microliter/nanoliter droplets. Following the analogy of digital electronics, this technology is referred to as "digital microfluidics" [12]. Compared to continuous-flow systems, digital microfluidics offer the advantage of dynamic reconfigurability and architectural scalability.

The level of system integration and the complexity of digital microfluidics-based biochips are expected to increase in the near future due to the growing need for multiple and concurrent bioassays on a chip [16]. However, shrinking processes, new materials, and the underlying multiple energy domains will make these biochips more susceptible to manufacturing defects. Moreover, some manufacturing defects are expected to be latent, and they may manifest themselves during field operation of the biochips. In addition, harsh operational environments may introduce physical defects such as particle contamination during field operation. Consequently, robust off-line and on-line test techniques are required to ensure system dependability as biochips are deployed for safety-critical applications such as field diagnostics tools to monitor infectious disease, and biosensors to detect biochemical toxins and other pathogens.

Although research in the design of digital microfluidicsbased biochips has gained considerable momentum in recent years $[12,15,16]$, only limited work has been reported thus far on biochip testing. A cost-effective test methodology for digital microfluidic systems was first described in [18]. Likely physical defects in such systems were analyzed and faults were classified as being either catastrophic or parametric. Faults are detected in [18] by electrically controlling and tracking the motion of test droplets. An optimal test planning method for the detection of catastrophic faults in digital microfluidic arrays was investigated in [19]. It is based on a graph model of the microfluidic array and a problem formulation based on Hamiltonian paths in a graph. An efficient concurrent testing method that interleaves test application with a set of bioassays was proposed in [20]. Reconfiguration and defect tolerance techniques for biochips were described in [17, 21].

Prior work on the testing of digital microfluidics-based biochips is based on invalid assumptions regarding the impact of certain defects on droplet flow. For example, a common defect seen in fabricated microfluidic arrays is a short-circuit between two adjacent electrodes [18]. It was assumed in [18-20] that this defect causes a droplet to be stuck at one of the two electrodes irrespective of the orientation of liquid flow. No attempt was made in prior work to experimentally validate this assumption. Experiments show however that the effect of this short-circuit defect on droplet flow depends on whether the droplet flow path is perpendicular to the two shorted electrodes or aligned with them. A test procedure for such defects should therefore not only test single cells as in [18-20], but it should also focus on pairs of cells and the traversal of droplets from one cell to all its neighbors. In addition, no systematic attempt has been made to relate defects to fault models and observable errors.

No attempt has been made in prior work to account for the hardware cost of droplet sources and sinks. The locations of droplet sources and sinks are determined manually, and the problem of determining these locations is not incorporated in the test planning problem. Moreover, as shown in [17, 21], digital microfluidic biochips offer dynamic reconfigurability to support defect tolerance, whereby groups of cells in a microfluidic array can be reconfigured to change their functionality in order to bypass defective cells. To facilitate this reconfiguration, we not only need a pass/fail test, but we also need to locate faulty cells. However, prior work has not addressed the issue of fault diagnosis in microfluidic arrays.

In this paper, we attempt to address the above issues for digital microfluidics-based biochips. First we relate some realistic defects to fault models and observable errors. We next set up an experiment to evaluate the manifestation of electrode shorts at the fluidic behavioral level. Motivated by the experimental results, we present a testing methodology based on graph theory to detect catastrophic faults, including those caused by electrode shorts. While this method can easily determine a test droplet flow path for off-line testing, we show that it can be extended to support on-line testing, whereby the test procedure is performed concurrently with a set of bioassays. This methodology can also automatically determine the location of test droplet sources/sinks to optimize the test plan. In addition, we investigate the problem of fault diagnosis. We apply this methodology to a real-life biochip performing multiplexed biochemical assays, and compare our results with the results reported in [20].

The organization of the remainder of the paper is as follows. Related prior work is described in Section 2. Next, fault modeling for digital microfluidic biochips is discussed in Section 3. Section 4 presents an experimental setup to evaluate the effect of electrode short defects. Next, a graph theory-based testing methodology is presented in Section 5. Both off-line and on-line testing methods are investigated. Diagnosis techniques to locate faulty cells in the microfluidic array are also discussed in 
this section. In Section 6, we evaluate the proposed test and diagnosis methodology by applying them to a biochip that can be used for point-of-care medical diagnostics. Finally, conclusions are drawn in Section 7.

\section{Prior Work}

MEMS is a relatively young field compared to microelectronics. The heterogeneity inherent in MEMS, resulting from the use of interacting mechanical and electronic devices, gives rise to many possible failure mechanisms and failure modes that are quite different from those in microelectronics. Thus efficient fault models and test generation methods for MEMS remain a major challenge. Recently, fault modeling and fault simulation for surfacemicromachined MEMS have been analyzed [2, 3, 9, 10]. In $[2,9]$, a comprehensive testing methodology for surface micromachined sensors has been presented. High-reliability and safety-critical markets for MEMS, e.g., accelerometers used in automobiles, are driving the integration of efficient built-in self-test and on-line monitoring functions. Design-for-manufacturing (DFM) and design-for-testability (DFT) methodologies have been incorporated in the design flow for MEMS [22].

However, test techniques for classical MEMS cannot be directly applied to microfluidic systems, since they differ in the underlying energy domains and in their working principles. The techniques and tools currently in use for the testing of classical MEMS (e.g., comb-drive microresonator) mainly aim at mechanical defects such as stiction; they do not handle fluids. Thus new testing techniques are required for microfluidics-based biochips. Very limited work has been reported in this area. Recently, fault modeling and fault simulation for continuous-flow microfluidic biochips have been proposed in $[6,7]$. Also, a DFT technique for microfluidic systems based on electro-osmotic flow has been discussed in [8].

The first attempt to address testing problem of dropletbased "digital" microfluidic biochips is described in [18]. In the proposed testing methodology, test stimuli droplets containing the normal conductive fluid (e.g., 0.1 M KCL) are released into a two-dimensional microfluidic array from on-chip reservoirs, and are guided through the system following the designed testing scheme. Both catastrophic and parametric faults are detected by electrically controlling and tracking the motion of these test stimuli droplets. This testing method is minimally invasive and easy to implement, thus it alleviates the need for expensive and bulky external testing devices.

The proposed unified detection mechanism consists of a simple RC oscillator circuit formed by the sink electrodes and the fluid between them as an insulator; see Fig. 1 [19]. The capacitance of this structure depends on the presence of the droplet since the filler medium and the droplet have distinct permittivities. By sensing the capacitance of this

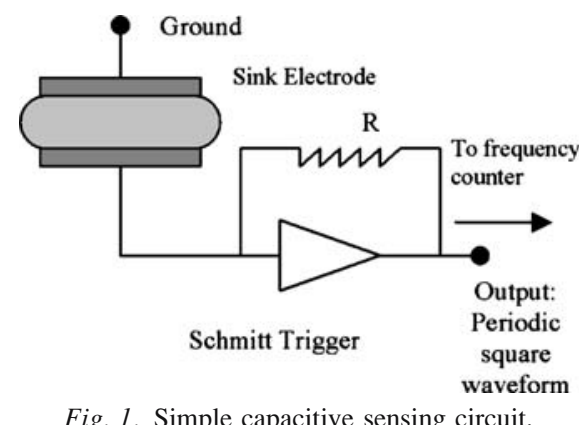

structure using a simple frequency counter, one can determine whether a droplet has reached the sink. This mechanism can be electronically implemented and easily integrated on-chip. In order to provide a unidirectional and unambiguous detection mechanism, the pass/fail criterion has to be determined based on the presence of the droplet at the sink electrode and this criterion should be applied for all test cases, i.e., the fault-free operation is associated with the presence of the droplet at the sink electrode and faulty operation with its absence.

In $[19,20]$, the test planning problem was formulated in terms of the graph partitioning and the Hamiltonian path problems from graph theory. The key idea underlying this optimization approach is to model the two-dimensional microfluidic array as a directed graph, and then partition it into non-overlapping subgraphs. In each subgraph, a Hamiltonian path from one source to one sink is determined as the flow path of test droplets such that each cell of a microfluidic array can be traversed. If one single cell becomes faulty, test droplet would be stuck during its transportation. The faulty status can be easily determined by observing the corresponding test points (i.e., sink electrodes connected with capacitive sensing circuits).

\section{Fault Modeling}

Like microelectronic circuits, a defective microfluidic biochip is said to have a failure if its operation does not match its specified behavior. In order to facilitate the detection of defects, fault models that efficiently represent the effect of physical defects at some level of abstraction are required. These models can be used to capture the effect of physical defects that produce incorrect behaviors in the electrical or fluidic domain. As described in [18], faults in digital microfluidic systems can be classified as being either catastrophic or parametric. Catastrophic faults lead to a complete malfunction of the system, while parametric faults cause degradation in the system performance. Table 1 lists some common failure sources, defects and the corresponding fault models for catastrophic faults in digital microfluidic biochips.

In addition, physical defects that cause parametric faults include geometrical parameter deviations. The deviation in insulator thickness, electrode length and 
Table 1. Some failure sources, corresponding defects, fault models, and observable errors in digital microfluidic biochips.

\begin{tabular}{|c|c|c|c|}
\hline Failure source & Defect & Fault model & Observable error \\
\hline $\begin{array}{l}\text { Excessive voltage applied to } \\
\text { electrode }\end{array}$ & Dielectric breakdown & $\begin{array}{l}\text { Short between the } \\
\text { droplet and the } \\
\text { electrode }\end{array}$ & $\begin{array}{l}\text { Droplet undergoes electrolysis, which prevents its further } \\
\text { transportation }\end{array}$ \\
\hline \multirow[t]{2}{*}{$\begin{array}{l}\text { Abnormal metal layer } \\
\text { deposition and etch variation } \\
\text { during fabrication }\end{array}$} & $\begin{array}{l}\text { Metal connection between } \\
\text { two adjacent electrodes }\end{array}$ & Electrode short & $\begin{array}{l}\text { A droplet resides in the middle of these two shorted } \\
\text { electrodes, and its transport along one or more directions } \\
\text { cannot be achieved }\end{array}$ \\
\hline & $\begin{array}{l}\text { Broken control wire to } \\
\text { control source }\end{array}$ & Electrode open & A failure in activating the electrode for droplet transport \\
\hline Particle contamination & $\begin{array}{l}\text { Fluidic high-impedance } \\
\text { between plates }\end{array}$ & Fluidic open & A droplet cannot move across the obstacle \\
\hline $\begin{array}{l}\text { Abnormal environment- } \\
\text { temperature variation during } \\
\text { operation }\end{array}$ & $\begin{array}{l}\text { Unexpected viscosity change } \\
\text { of fluids (droplet and filler } \\
\text { medium) }\end{array}$ & Fluidic open & $\begin{array}{l}\text { A droplet cannot move due to the increased friction at the } \\
\text { droplet/medium interface }\end{array}$ \\
\hline
\end{tabular}

height between parallel plates may exceed their tolerance value during fabrication. Note that, even though the methodology described in this paper is applied to only catastrophic faults, it can easily be extended for the detection of parametric faults based on the unified detection mechanism proposed in [18].

It is evident that catastrophic faults can lead to a complete cessation of droplet transportation. However, there exist differences between their corresponding erroneous behaviors. For instance, to test for the electrode-open fault, it is sufficient to move a test droplet from any adjacent cell to the faulty cell. The droplet will always be stuck during its motion due to the failure in charging the control electrode. On the other hand, if we move a test droplet across the faulty cells affected by an electrode-short fault, the test droplet may or may not be stuck depending on its flow direction. In the next section, we design a defect-oriented experiment to evaluate the behavioral impacts of electrode-short faults.

\section{Defect-Oriented Experiments}

\subsection{Microfluidic Biochip Description}

The microfluidic biochip discussed in this paper is based on the manipulation of microliter-nanoliter droplets using the principle of electrowetting-on-dielectric (EWOD) [11,
12]. Electrowetting refers to the modulation of the interfacial tension between a conductive fluid and a solid electrode coated with a dielectric layer by applying an electric field between them. An imbalance of interfacial tension is created if an electric field is applied to only one side of the droplet; this tension gradient forces the droplet to move.

The basic cell of an EWOD-based digital microfluidic biochip consists of two parallel glass plates, as shown in Fig. 2. The bottom plate contains a patterned array of individually controllable electrodes, and the top plate is coated with a continuous ground electrode. The control electrodes in the bottom plate are coated with a dielectric insulator, e.g., parylene $\mathrm{C}$, for insulation. A hydrophobic thin film is also added to the top and bottom plates to decrease the wettability of the surface and to add capacitance between the droplet and the control electrode. The droplet containing biochemical samples and the filler medium, such as the silicone oil, are sandwiched between the plates; the droplets travel inside the filler medium.

In order to move a droplet, a control voltage is applied to an electrode adjacent to the droplet (e.g., electrode 3 in Fig. 2) and at the same time the electrode just under the droplet (e.g., electrode 2 in Fig. 2) is deactivated. Thus, the charge in the droplet/insulator interface that is accumulated over the activated electrode results in an interfacial tension gradient, which consequently causes droplet transport. By varying the electrical potential along
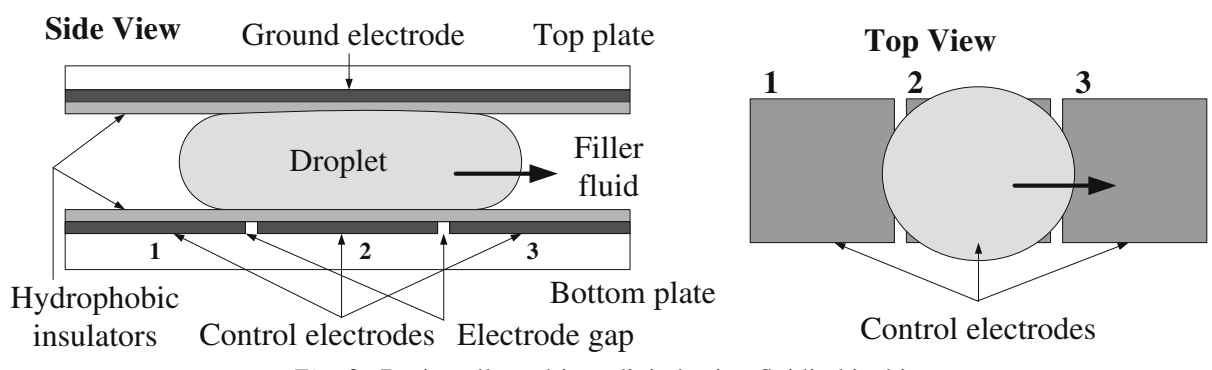

Fig. 2. Basic cell used in a digital microfluidic biochip. 

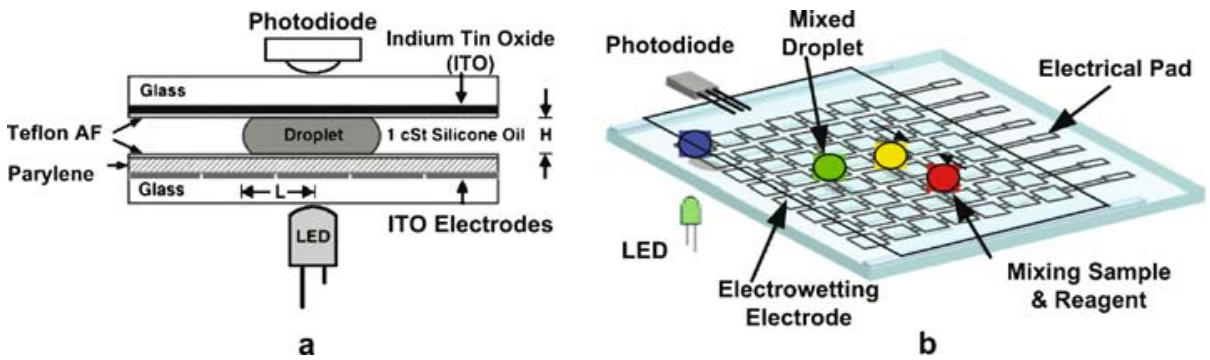

Fig. 3. Schematic of a digital microfluidic biochip used for colorimetric assays: a basic cell; $\mathbf{b}$ top view of microfluidic array.

a linear array of electrodes, microliter/nanoliter-volume droplets can be transported along this line of electrodes. The velocity of the droplet can be controlled by adjusting the control voltage $(0-90 \mathrm{~V})$, and droplets have been observed to move with velocities up to $20 \mathrm{~cm} / \mathrm{s}$ [12]. Furthermore, based on this principle, droplets can be transported freely to any location on a two-dimensional array without the need for micropumps and microvalves that are required in continous-flow systems.

Using a two-dimensional microfluidic array, many common operations for different bioassays can be performed, such as sample movement (transport), temporary sample preservation (store), and the mixing of different samples (mix). For instance, the store operation is performed by applying an insulating voltage around the droplet. The mix operation is used to route two droplets to the same location and then turn them about some pivot points. Note that these operations can be performed anywhere on the array, whereas in continuous-flow systems they must operate in a specific micromixer or microchamber. This property is referred to as the reconfigurability of a digital biochip. The configurations of the array, i.e., the droplet transport routes and their rendezvous points, are programmed into a microcontroller that controls the voltages of electrodes in the array.

The in vitro measurement of glucose and other metabolites, such as lactate, glutamate and pyruvate, is of great importance in clinical diagnosis of metabolic disorders. A colorimetric enzyme-kinetic glucose assay has been recently demonstrated in lab experiments on a digital microfluidic biochip [13-15]. This biochip uses a digital microfluidic array, which moves and mixes droplets containing biochemical samples and reagents, and an integrated optical detection system consisting of a LED and a photodiode; see Fig. 3 [13-15].

\subsection{Experiment Design}

To evaluate the effect of an electrode short on microfluidic behavior, we design an experiment using a $2 \times 4$ microfluidic array as shown in Fig. 4(a). This experiment includes two steps. First, we impose the condition that two electrodes adjacent in the $X$-direction, e.g., electrode 6 and 7 in Fig. 4(b), are shorted. A horizontal flow path, e.g., $5 \rightarrow 6 \rightarrow 7 \rightarrow 8$, is used to guide a test droplet across the shorted cells. The effect of the short between two adjacent electrodes can be simulated by simultaneously changing the voltages on these two electrodes. In the second step, two electrodes adjacent in the $Y$-direction, e.g., electrode 2 and 6 in Fig. 4(c) are considered to be shorted. As in the first step, a test droplet traverses the faulty cell (electrode 6) following a flow path in the $X$ direction (e.g., $5 \rightarrow 6 \rightarrow 7$ ). For both steps, we use optical devices such as CCD cameras to visually inspect if the test droplet is stuck during its transportation.

\subsection{Chip Fabrication}

The $2 \times 4$ microfluidic array used in the experiment was fabricated using standard microfabrication techniques. The detailed fabrication process is described in [11]. The control electrodes in the bottom glass plate are formed by a $200 \mathrm{~nm}$ thick layer of chrome, which is further coated with a layer of Parylene C $(800 \mathrm{~nm})$ as a dielectric insulator. This microfluidic array uses a $1.0 \mathrm{~mm}$ electrode pitch size. A layer of optically transparent indium tin oxide (ITO) in the top glass plate is used as the continuous ground electrode. In addition, a 50-nm-thick film of Teflon AF 1600 is added as the hydrophobic coating on both the top and the bottom plates. The $600 \mu \mathrm{m}$ gap between the top and bottom plates is set using a glass spacer.

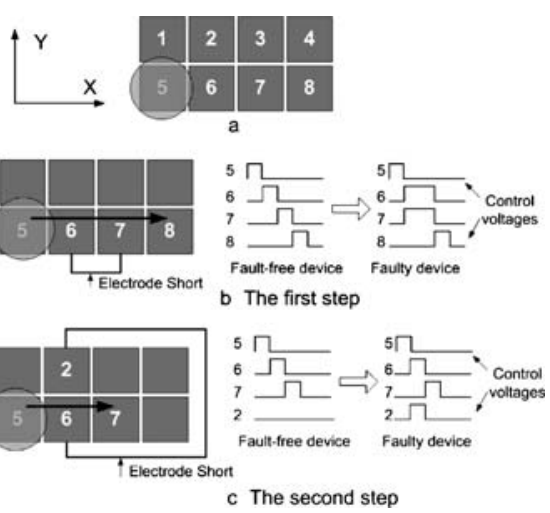

Fig. 4. Design of an experiment to study microfluidic behavior in the presence of the electrodeshort fault. 


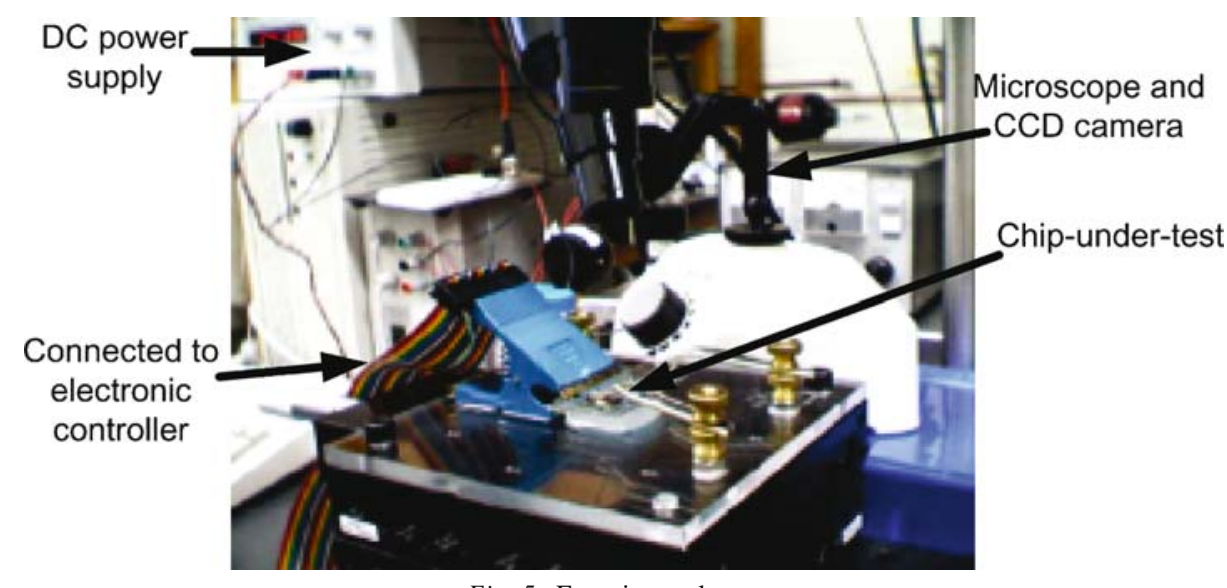

Fig. 5. Experimental setup.

\subsection{Experimental Setup}

The experimental setup for testing the $2 \times 4$ microfluidic array is shown in Fig. 5. The chip-under-test was mounted on a custom-assembled platform. We use a custom-made electronic unit to independently control the voltages of each control electrode in the array by switching them between ground and a DC actuation voltage. In our experiments, the actuation voltage was set at $50 \mathrm{~V}$. A $1-\mu \mathrm{l}$ test droplet containing $0.1 \mathrm{M} \mathrm{KCL}$ was dispensed onto the chip using a micropipettor; the filler fluid medium, i.e., $1 \mathrm{cSt}$ silicone oil was introduced after droplet dispensing. Images of droplet transportation during the experiment were obtained with an industrial microscope (VZM 450i, Edmund Industrial Optics) and a color CCD camera (Sony XC-999). Images were either captured directly to a PC using a frame grabber (MicroDC30, Pinnacle Systems) or were video-recorded with a super-VHS videocassette recorder (JVC-S4600).

\subsection{Results and Analysis}

In the first step of the evaluation experiment, we let a test droplet move through two electrodes that are adjacent in the $X$-direction. As indicated before, these two electrodes are effectively shorted by setting them to identical voltages. A droplet aligns itself with the charged electrode to maximize the area of overlap and therefore the electrostatic energy stored in the effective capacitors between the droplet and the electrode. Thus the test droplet resides around the middle of two shorted electrodes as shown in Fig. 6. Since there is no overlap between this droplet and neighboring electrode (i.e., electrode 8), the test droplet cannot be further moved to electrode 8; it is stuck between electrode 6 and electrode 7 in the experiment.

The second step of the experiment is to investigate what happens when there is a short between two electrodes that are adjacent in the $Y$-direction. Interestingly, our experiment shows that in this case, the test droplet can still move across electrode 6 , even though this electrode is shorted with electrode 2; see Fig. 7. We can explain this phenomenon on the basis of the fact that there still exists sufficient overlap between the test droplet and electrode 7, even though the droplet tends to move towards the middle of electrodes 6 and 2. Thus, the test droplet is not stuck if it follows the test plan $5 \rightarrow 6 \rightarrow 7$.

The above experimental results provide useful insights on how testing should be carried out for microfluidic arrays. We find that electrode short faults lead to an error only when the droplet flow path is aligned with the orientation of the electrode shorts. In addition to electrode short, there exist other physical defects that lead to similar erroneous behavior. For example, particle contamination between two adjacent cells also produces an error under specific droplet flow paths. In order to detect these defects, a test plan should guide the test droplet to move from a cell in the array to all its neighbors.

These experimental results also highlight a major deficiency of prior work on the testing of microfluidic arrays $[19,20]$. The previous approaches map the droplet flow path problem to that of finding a Hamiltonian path in a graph model of the array. In other words, the test droplet is routed through the array such that it visits every cell exactly once. While this approach guarantees the detection of faults involving only one electrode or cell, it is not sufficient to detect electrode-short and fluidic-open faults that affect two adjacent electrodes. This is highlighted in the next section.
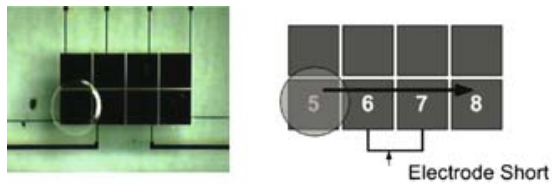

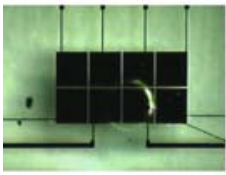

a

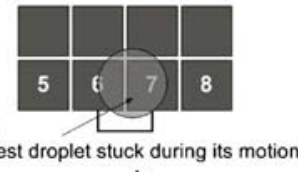

b
Fig. 6. Experimental results and analysis for the first step. 

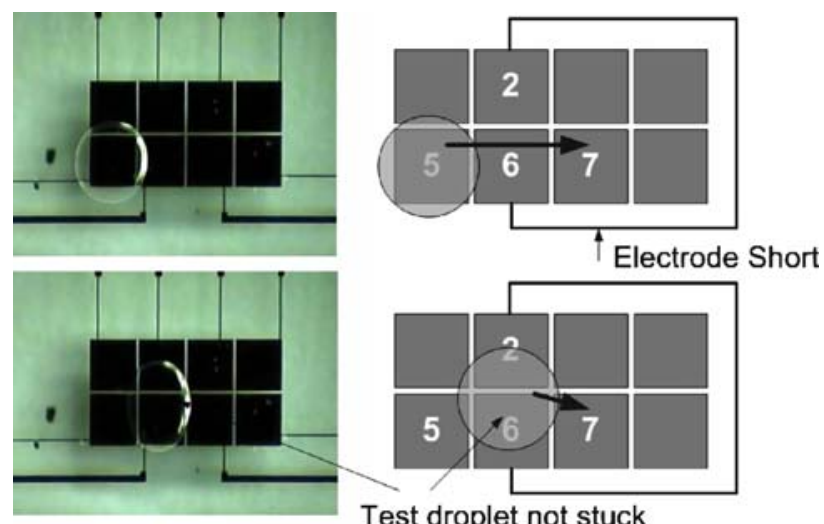

Test droplet not stuck

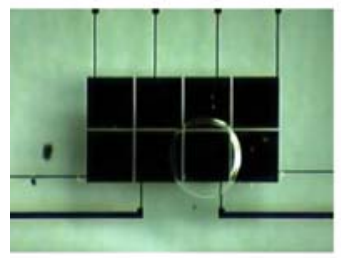

a

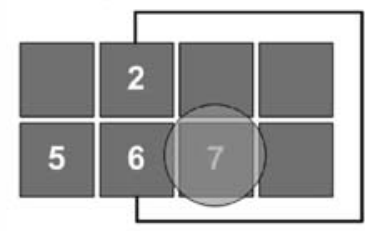

b
Fig. 7. Experimental results and analysis for the second step.

\section{Testing and Diagnosis}

The "edge-dependent" nature of some defects (e.g., electrode shorts), as seen in Section 4, indicates that test planning methods proposed in $[19,20]$, which are based on the notion of the Hamiltonian path from graph theory, are not sufficient for fault detection. For example, in Fig. 4(c) the test droplet path $5 \rightarrow 6 \rightarrow 7 \rightarrow 8 \rightarrow 4 \rightarrow 3 \rightarrow 2 \rightarrow 1$ fails to detect an electrode short fault between electrodes 2 and 6 , even though this Hamiltonian path-based flow visits each cell exactly once. Thus, a new test planning method is required to deal with this problem. Since this type of defect can be introduced into microfluidic biochips not only during fabrication (e.g., electrode shorts due to manufacturing problems), but also during in-field operation (e.g., due to particle contamination and electrode metal migration), both off-line and on-line testing techniques are necessary. In addition, to support defect tolerance based on reconfiguration, a diagnosis technique is needed to locate candidate fault sites in a microfluidic array that is deemed to be faulty by the testing procedure.

\subsection{Off-Line Testing}

Test droplets are first dispensed onto the microfluidic array from the droplet source (i.e., on-chip reservoir and dispensing port). They are then routed through the biochip-under-test, i.e., traversing all the cells and cell boundaries. If there exists a catastrophic fault on the chip, the test droplet gets stuck at an intermediate point. Otherwise, it is eventually guided back to the droplet sink. The sink electrode is connected to a capacitive detection circuit that can determine the presence of the test droplet [18]. In this way, we can easily determine the faulty or fault-free status of the microfluidic biochip from the electrical output of the detection circuit.

We formulate the test planning problem in terms of the Euler circuit and Euler path problems from graph theory [13]. The key idea underlying this approach is to model the digital microfluidic array under test as an undirected graph, and then "eulerize" this graph. On the basis of Euler's theorem [13], a flow path for the test droplet can be easily obtained, which allows us to detect shorts between any two directly adjacent electrodes in the array.

First, we model the array of microfluidic cells using an undirected graph $G=(V, E)$ where the set of vertices $V$ represents the set of microfluidic cells in the array, and each edge is an unordered pair of vertices. The edge $\{u, v\} \in E$ if and only if vertex $u$ and vertex $v$ represent two directly adjacent microfluidic cells. Figure 8(a) shows an example of the graph model for a $5 \times 5$ microfluidic array.

An Euler path in a graph $G$ is defined as a path that traverses all the edges of $G$ exactly once [23]. Similarly, an Euler circuit is a cycle that traverses all the edges of the graph exactly once. We know from [23] that an undirected graph has an Euler circuit if and only if it is connected, and each vertex has even degree. Moreover, an undirected graph has an Euler path if it is connected and has exactly two vertices of odd degree. The Euler path must start at one of the odd-degree vertices and must end at the other odd-degree vertex [23].

Euler's theorems give us the means for finding efficient ways in which to traverse all the edges of an undirected graph. However, we notice that a graph model of a microfluidic array usually has more than two vertices of odd degree. Thus we have to retrace some of the edges in order to traverse all edges at least once. In order to apply existing Euler cycle/path algorithms, we need to convert some or all the vertices of odd degree to even degree by adding additional edges. The process of eliminating odd

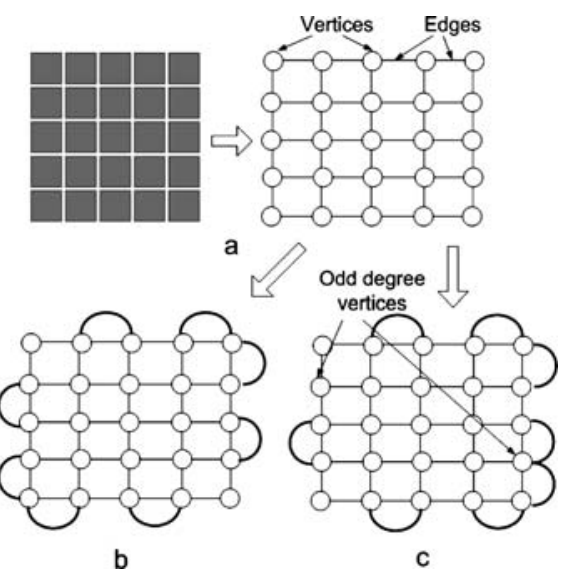

Fig. 8. (a) Graph model for a $5 \times 5$ microfluidic array; (b) eulerized graph containing an Euler circuit; (c) eulerized graph containing an Euler path. 
degree vertices by adding additional edges is called eulerizing the graph. There are two different ways for eulerizing the graph model of a microfluidic array, depending on whether an Euler circuit or an Euler path is desired. For example, as shown in Fig. 8(b), there exists an Euler circuit in the eulerized graph model for a $5 \times 5$ microfluidic array since each vertex becomes to be even degree. On the other hand, another eulerized graph in Fig. 8(c) contains an Euler path starting from one odd-degree vertex, e.g., cell $(2,1)$ and ending at another odd-degree vertex, e.g., cell $(4,5)$.

Although both these eulerizing methods can provide an edge tour as the feasible flow path of a test droplet, we use the first method (i.e., to find an Euler circuit) here. There are two main reasons for this choice. First, in the second eulerizing method we must use the node with odd degree as the starting or the ending point. Thus, to find an Euler path between another pair of cells, a different eulerized graph is required. In contrast, since any vertex can be used as the start and end point of an Euler circuit, we can locate the test droplet source/ sink adjacent to any boundary cell using the same eulerized graph in the first method. Thus, this method is especially suitable when we try to determine the optimal location of droplet sources and sinks. Second, we are motivated by considerations of physical implementation. If we merge the test droplet source and sink, i.e., connect the electrode of the dispensing port to the capacitive detection circuit, it not only reduces the area overhead of the test hardware, but it can also conserve the liquid volume of on-chip reservoir by recycling test droplets. This reduces the cost of manual maintenance. This feature is especially desirable for in-field testing.

Using the selected Eulerizing method, a graph model for the microfluidic array under test is modified to $G^{\prime}=\left(V, E^{\prime}\right)$, where the new set of edges $E^{\prime}$ includes all edges from $E$ as well as the additional edges. The following theorem quantifies the number of additional edges that are necessary.

Theorem 1 The minimum number of additional edges $N a$ required to eulerize an $m \times n$ microfluidic array such that an Euler circuit exists in the corresponding graph, is given by:

$$
N a=\left\{\begin{array}{l}
m+n-4, \text { if } m \text { and } n \text { are even; } \\
m+n-2, \text { otherwise. }
\end{array}\right.
$$

Proof: Since in an $m \times n$ array all internal vertices have even degree, i.e., 4 , we only need to add additional edges to the boundary vertices. Then this theorem can easily be proven using three different cases: (1) if $m$ and $n$ are both odd, $N a=2\left\lfloor\frac{m-1}{2}\right\rfloor+2\left\lfloor\frac{n-1}{2}\right\rfloor=m+n-2$; (2) if $m$ or $n$ is even and another one is odd, $N a=\left\lfloor\frac{m-1}{2}\right\rfloor+\left\lfloor\frac{m}{2}\right\rfloor+\left\lfloor\frac{n-1}{2}\right\rfloor+$ $\left\lfloor\frac{n}{2}\right\rfloor=m+n-2$; (3) if $m$ and $n$ are both even, $N a=$ $2\left\lfloor\frac{m-1}{2}\right\rfloor+2\left\lfloor\frac{n-1}{2}\right\rfloor=m+n-4$.
Based on Theorem 1, we find that the total number of edges of an eulerized graph model $G^{\prime}=\left(V, E^{\prime}\right)$ for an $m \times n$ microfluidic array is as follows.

$$
\begin{aligned}
N\left(E^{\prime}\right)=N(E) & +N a=(2 m n-m-n)+N a \\
& =\left\{\begin{array}{l}
2 m n-4, \text { if } m \text { and } n \text { are even } \\
2 m n-2, \text { otherwise }
\end{array}\right.
\end{aligned}
$$

We next define the length of a time slot to be equal to the time during which a test droplet moves from one cell to an adjacent one. Thus, the total testing application time is $N\left(E^{\prime}\right)$ time slots, if a test droplet follows an Euler circuit-based path.

To find an Euler circuit in the eulerized graph, we use the well-known Fleury's algorithm; its pseudocode is shown in Fig. 9 [23]. The advantage of this algorithm is that since it is a real-time search algorithm, it can be easily modified to handle both multiple test droplets and the concurrent testing problem.

The identification of an edge as a bridge, i.e., cut edge, ${ }^{1}$ in Fleury's algorithm can be achieved by applying depth-first search to check the connectivity of the untested part of the graph [1]. Although it works well for a microfluidic array of modest size, its complexity is $O(n+e)$, where $n$ and $e$ are the number of vertices and edges in the part of an undirected graph that has not been visited, respectively. This amounts to high computation cost because of the need for iterative connectivity checking during the search for an Euler circuit. Therefore, we modify Fleury's algorithm by replacing bridge checking with a probabilistic search procedure based on some simple rules of complexity $O(1)$. We probabilistically select the edge to visit. The probability assignment is based on some simple rules, which can be used as guidelines to find Euler circuits; some of these rules are listed as follows:

(1) Do not use an edge to go to a vertex unless there is another edge available to leave that vertex (except for the last step). An example of probability assignment based on this rule is shown in Fig. 10(a).

(2) An edge that belongs to a loop is not a bridge. Note that if there exist two "not visited' edges between two adjacent vertices, they form a loop. Thus, we can select one such edge with a higher probability compared to other edges; see Fig. 10(b).

Although this rule-based search cannot guarantee the identification of an Euler circuit in one run, an appropriate number of simulation runs can easily lead to the desired result. This method is scalable to large problem sizes. In addition, the starting point, i.e., the location of droplet source and sink, can be selected at random, which is especially important for multiple test droplets and for

\footnotetext{
${ }^{1}$ A cut edge (bridge) of a graph $G$ is an edge whose removal disconnects $G$.
} 


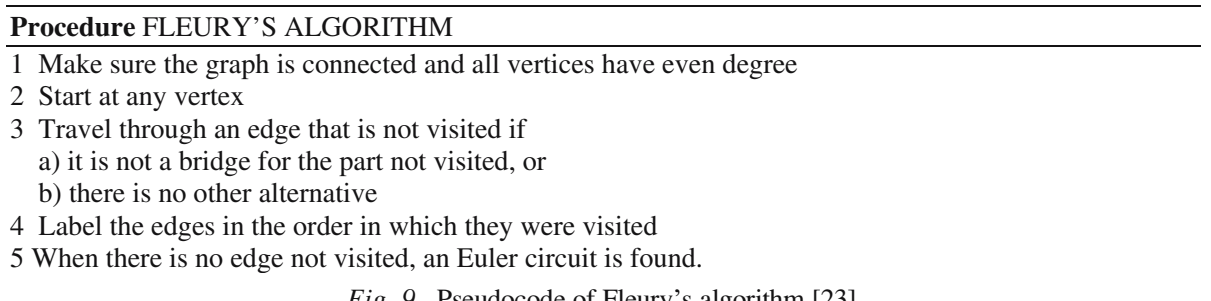

Fig. 9. Pseudocode of Fleury's algorithm [23].

concurrent testing. The pseudocode of this probabilistic modified Fleury's algorithm (PMF) is shown in Fig. 11.

The Euler circuit-based method can be further extended to find a test schedule for more than one test droplet. We first partition the graph model of a microfluidic array into subgraphs, and then eulerize them individually such that there exists an Euler circuit in each subgraph. In this way, multiple test droplets can perform the edge-tour testing simultaneously in different parts of the microfluidic array. The total testing application time is the maximum of the testing time for any of these subgraphs. This leads to the reduction of the testing time at the expense of test hardware overhead, corresponding to multiple droplet sources/sinks. Figure 12 shows an example of two test droplets that are applied to a $5 \times 5$ microfluidic array. The testing time can be reduced significantly, i.e., from 48 time slots to 28 time slots. Note that there exist overlaps between the different subgraphs in order to cover all edges in the graph, as shown in Fig. 7. However, we must not allow two test droplets to traverse an edge at the same time. In addition, an important constraint arising from fluidic considerations is that a droplet should never be in a cell directly adjacent or diagonally adjacent to another droplet; otherwise, these two droplets will mix together. This restriction increases the complexity of test planning problem and it may introduce waiting time (stall cycles) for some test droplets. The proposed PMF algorithm can be easily modified to solve the above problem. To ensure that fluidic constraints are satisfied, we assign a random (but distinct) priority to each test droplet; the test droplet movements are planned in prioritized order, whereby in each time step the test droplet with higher priority is scheduled first, and the droplet with lower priority attempts to avoid the droplet with higher priority.

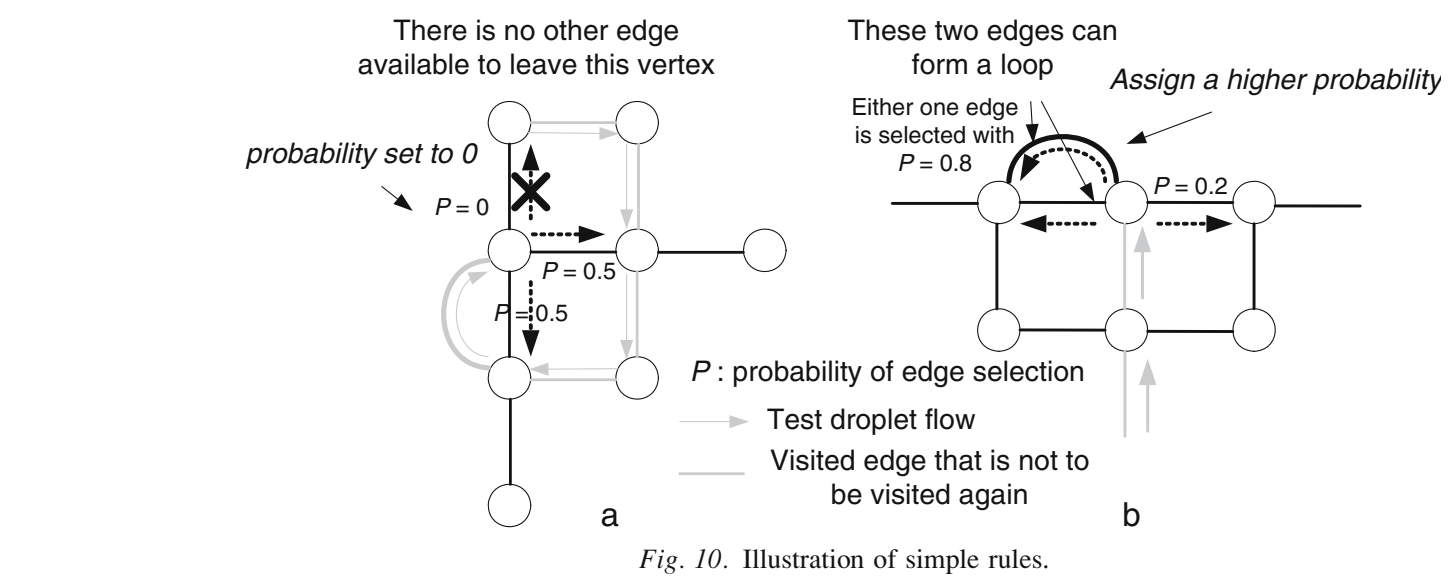

\subsection{On-Line Testing}

Some cells in a digital microfluidic biochip may be rendered faulty during in-field operation. Therefore, on-line concurrent testing, which allows testing and normal bioassays to run simultaneously on a chip, can play an important role in alerting the user to an unpredictable faulty status.

We can easily modify the PMF algorithm to derive a test plan that support on-line concurrent testing. We assume that the schedule of a bioassay performed on the microfluidic biochip is known a priori, e.g., using methods described in [16]. The goal of a desirable test plan is to avoid conflicts with the normal assay operation while traversing all the edges in the array. Thus, an additional evaluation step is added to the search procedure in the PMF algorithm, i.e., in each time step we need to check the other endpoint (vertex) of each candidate edge. If this vertex represents the cell that is occupied by the assay operation at this time slot or adjacent to an assay droplet, the corresponding edge cannot be visited. If no edges are available at this time step, the test droplet must wait at the current cell until there is an available edge to visit. The total concurrent testing time equals Euler tour time, i.e., $N\left(E^{\prime}\right)$ time slots, plus the waiting time. Different locations of test droplet sources and sinks can affect the on-line testing time. By randomly selecting the starting point, the PMF algorithm attempts to find the best location of test droplet sources and sinks to minimize the testing time. Moreover, as in off-line testing, multiple test droplets can be applied to reduce the testing time, whereby each test droplet is guided to traverse the partition and also does not conflict with the bioassay in this region. 


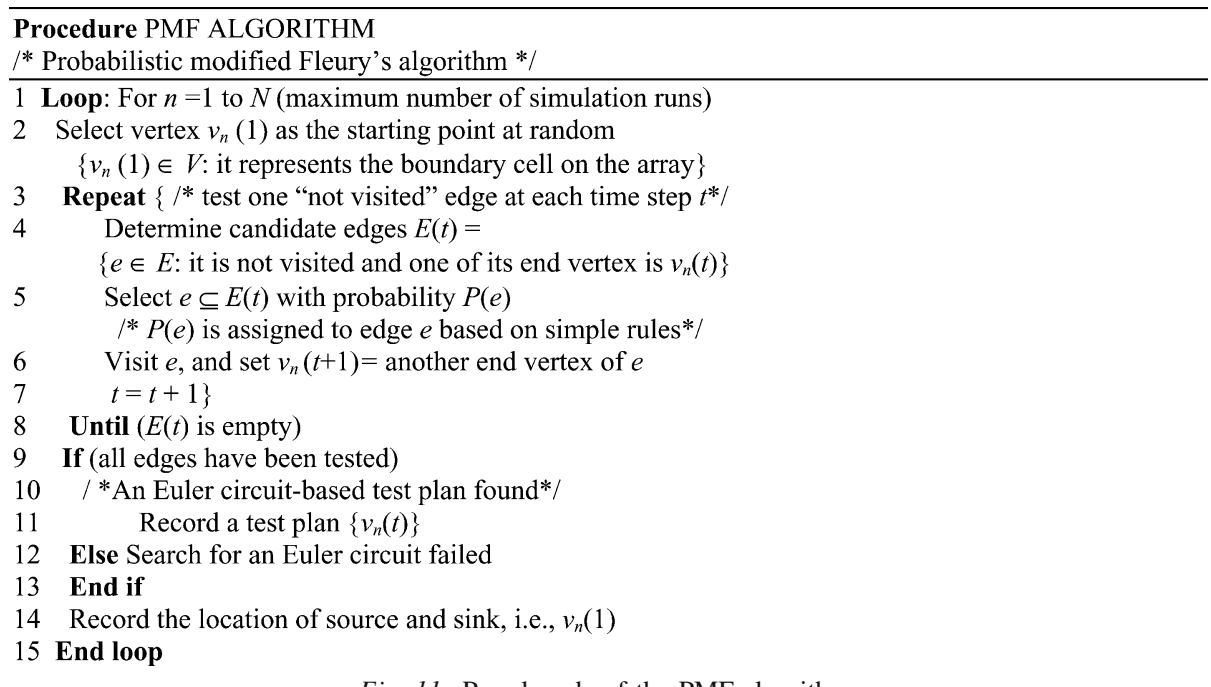

Fig. 11. Pseudocode of the PMF algorithm.

\subsection{Diagnosis}

In order to increase the reliability and system lifetime of digital microfluidic biochips, defect tolerance based on reconfiguration can be used to bypass faulty cells [17, 21]. We implement the diagnosis procedure using multistep and adaptive Euler circuit-based testing methods. In each step, we divide the candidate faulty region into two partitions, and then test each partition to determine whether it is a candidate faulty region. Under single fault assumption [21], we can simply check either one binary partition to determine the faulty candidate region. By using a series of adaptive testing steps, we can eventually determine the location of candidate faulty cells. Assume that such a diagnosis procedure includes a series of testing steps, i.e., $T_{1}, T_{2}, \ldots T_{k}$, where $T_{i}(i=1 \sim k)$ denotes an Euler circuit-based traversal of the candidate faulty region at step $i$, and the final testing step $T_{k}$ is to traverse a $2 \times 2$ array, i.e., the minimum candidate faulty region that can be located by Euler circuit-based approach. The number of steps $k$ for a given microfluidic array size is given by using the following theorem.

Theorem 2 To locate any single fault (including electrode-short faults) in an $m \times n$ microfluidic array $(m, n>2)$, the number of Euler circuit-based testing steps $k$ in the proposed diagnosis scheme is $k=\left\lceil\log _{2}(m-1)\right\rceil+$ $\left\lceil\log _{2}(n-1)\right\rceil$.

Proof: We can prove this theorem by using the two-phase partitioning schemes. In the first phase, we split the array in half with a cutting line in the $Y$-direction (North-South). The binary partition is recursively applied until each partition contains only one edge in the row of the corresponding subarray. The number of steps in recursive binary partitioning is $\left\lceil\log _{2}(n-1)\right\rceil$. Next, a similar partitioning scheme is applied to the $m \times n$ array with a cutting line in the $X$-direction, until each partition only has one edge in the column; the number of binary partitioning steps is $\left\lceil\log _{2}(m-1)\right\rceil$ in this phase. Through these two phases, we are able to locate any single fault to a minimum candidate faulty region. The total number of partitioning steps is $\left\lceil\log _{2}(m-1)\right\rceil+\left\lceil\log _{2}(n-1)\right\rceil$, which is a sufficient number of adaptive testing steps to locate any single fault. Thus $k=\left\lceil\log _{2}(m-1)\right\rceil+\left\lceil\log _{2}(n-1)\right\rceil$.

We denote the time needed for each testing step $T_{i}$ by $\operatorname{Tt}\left(T_{i}\right)$; it includes the Euler traversal time in the candidate faulty region described in Section 5.1, and the droplet

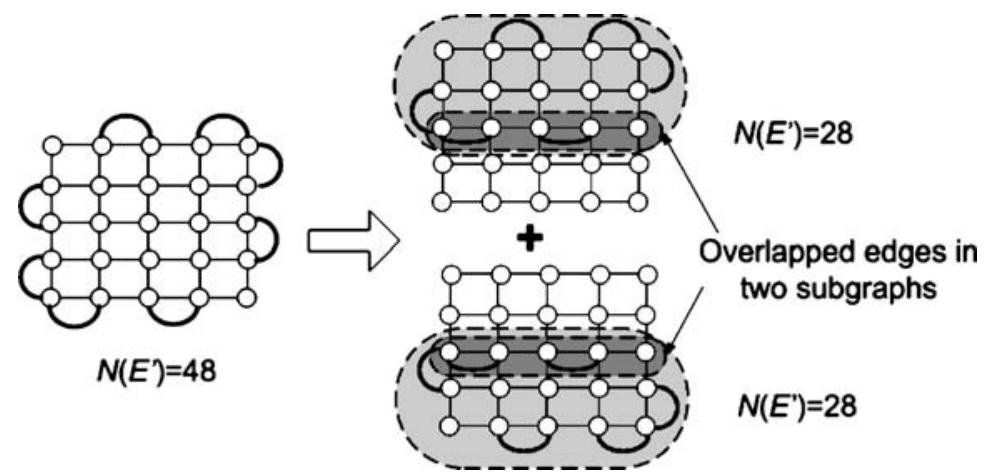

Fig. 12. Application of two test droplets to a $5 \times 5$ microfluidic array. 

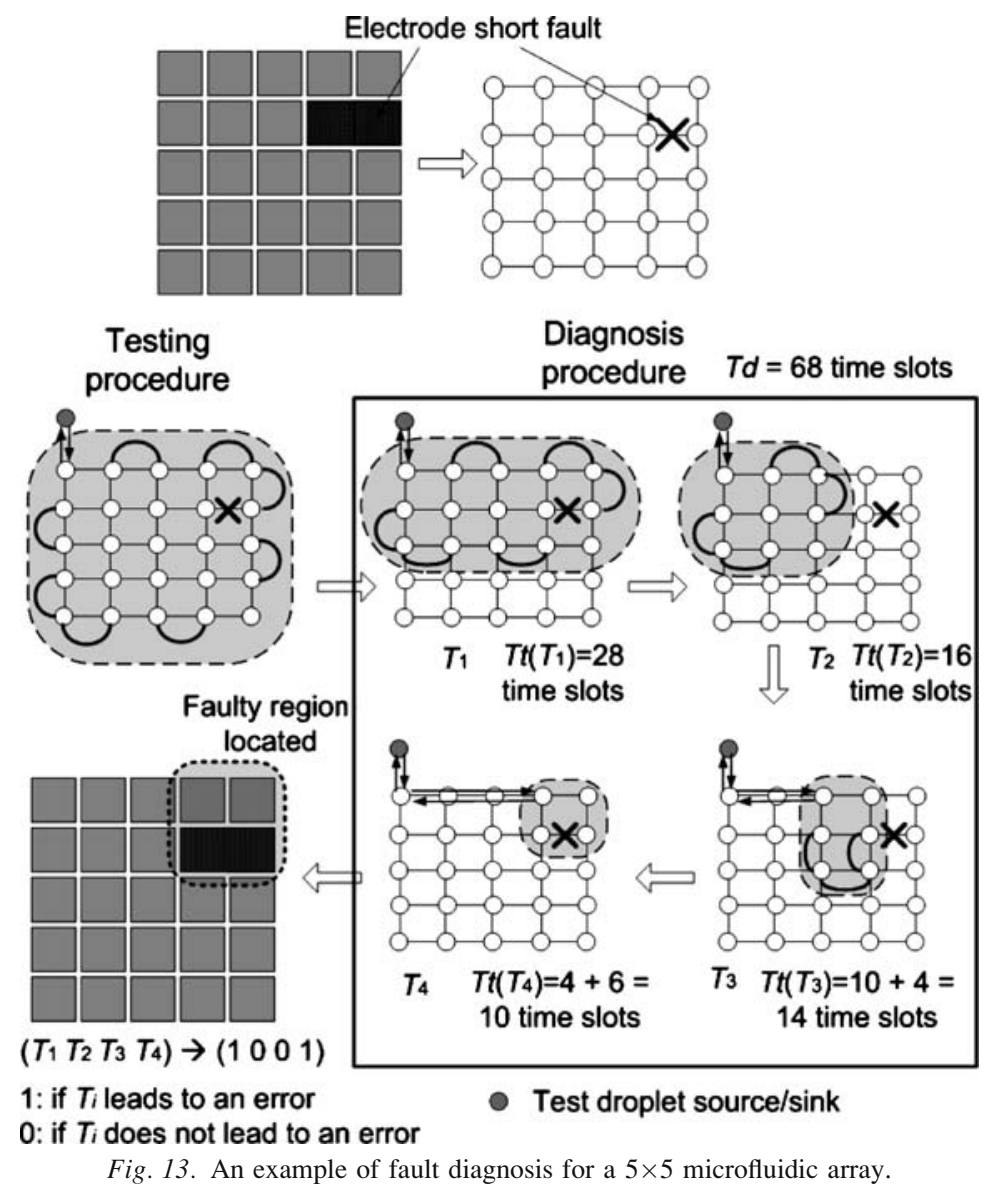

transportation time between the droplet source/sink and the testing region (if droplet source and sink are not adjacent to this testing region). Thus, the total diagnosis time $\mathrm{Td}$ is $T d=\sum_{i=1}^{k} T t\left(T_{i}\right)$.

Figure 13 illustrates the adaptive diagnosis procedure for an array with an electrode-short fault. Based on the single fault assumption, we can easily locate the faulty region caused by the electrode-short fault through a series of testing steps, i.e., $T_{1}-T_{4}$. If some bioassay operations are scheduled in this region, they must be remapped to other faulty-free regions on the microfluidic array to avoid erroneous assay results. This diagnosis method can locate not only single faults, but it can also easily be extended to locate multiple faults by using multiple test droplet sources and sinks.
Furthermore, a comprehensive testing and diagnosis procedure can be developed for digital microfluidic biochips. As digital microfluidic biochips become widespread in safety-critical biochemical applications, the reliability of these systems will emerge as a critical performance parameter. These systems need to be tested adequately not only after fabrication (off-line), but also continuously during in-field operation (on-line). Once the testing procedure determines the faulty status of biochips, the operation of the normal bioassay is stopped. Diagnosis techniques are applied to determine the location of faulty region. Then reconfiguration techniques are applied to tolerate operational faults; the biochip is redesigned with the help of the proposed system-level design automation tools.

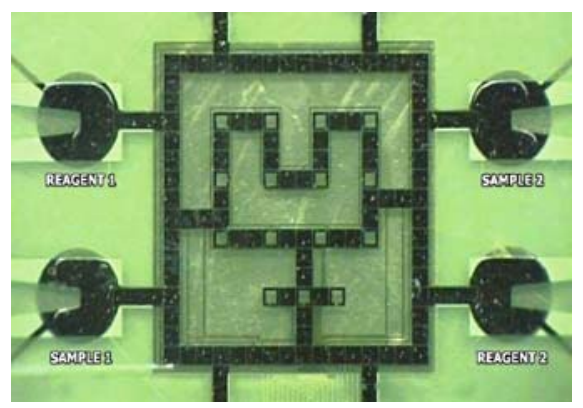

Fig. 14. Fabricated microfluidic array used for multiplexed bioassays [20].

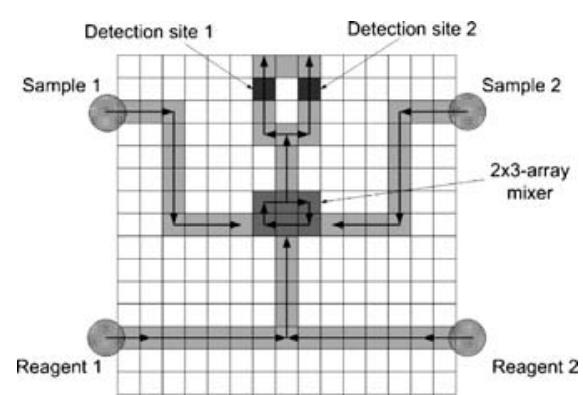

Fig. 15. A $15 \times 15$ microfluidic array used for multiplexed bioassays. 
Table 2. Schedule of multiplexed biomedical assay.

\begin{tabular}{ll}
\hline Time (s) & Operation \\
\hline 0 & Sample 2 and reagent 2 start to move towards the mixer \\
0.8 & Sample 2 and reagent 2 begin to mix together and turn around in the $2 \times 3$ array \\
6.0 & (1) Sample 1 and reagent 1 start to move towards the mixer \\
& (2) Sample 2 and reagent 2 continue the mixing \\
6.8 & (1) Sample 2 and reagent 2 finish the mixing and product 2 leave the mixer to optical detection location 2 \\
12.8 & (2) Sample 1 and reagent 1 begin to mix in $2 \times 3$ array mixer \\
19.8 & (1) Sample 1 and reagent 1 finish the mixing and product 1 leave the mixer to the optical detection location 1 \\
25.8 & (1) Product 2 finishes optical detection and leaves the array to the waste reservoir \\
& (2) Product 1 continues the absorbance detection 1 finishes optical detection and leaves the array to the waste reservoir. One procedure of the multiplexed biomedical assay ends.
\end{tabular}

Sample 1 and Reagent 1 are for glucose assay; Sample 2 and Reagent 2 are for lactate assay.

\section{Real-Life Application}

In this section, we use the real-life application example from [20], i.e., multiplexed glucose assay and lactate assay, to illustrate how Euler circuit-based method can be used for off-line testing, on-line testing and diagnosis in digital microfluidic biochips.
The glucose assay performed on digital microfluidic biochips is based on Trinder's reaction, a colorimetric enzyme-based method. In addition to glucose assays, the detection of other metabolites such as lactate, glutamate and pyruvate using digital microfluidics has also been demonstrated recently $[1,13,15]$. Furthermore, all these assays can be integrated to form a set of multiplexed
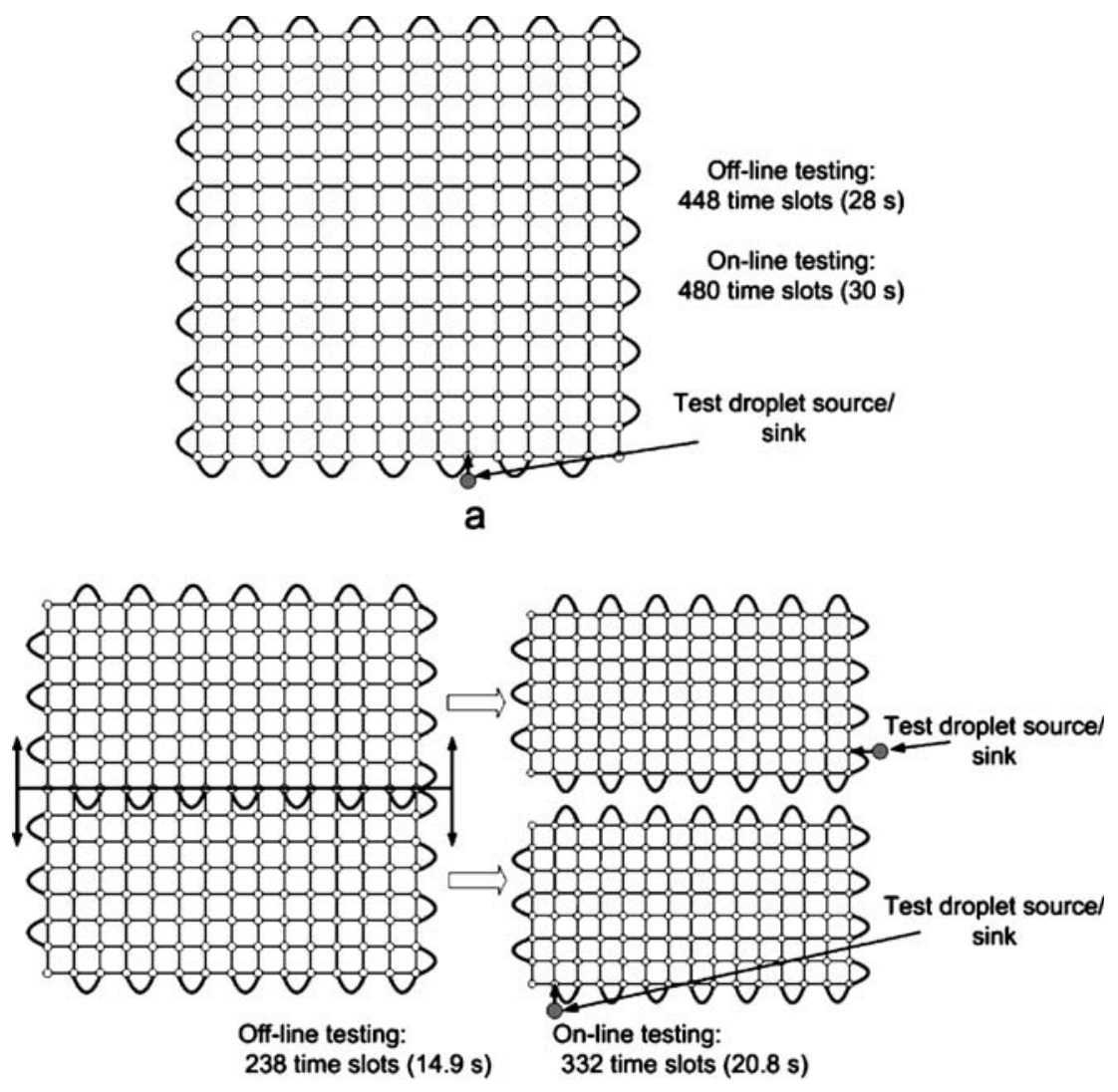

b

Fig. 16. Testing of a $15 \times 15$ microfluidic array: a Eulerized graph for the application of the single test droplet; b Partitions and eulerized graphs for the application of two test droplets. 
bioassays that are performed concurrently on a microfluidic platform. Figure 14 illustrates a fabricated microfluidic biochip prototype used for multiplexed bioassays [5]. For example, Sample 1 can be assayed for glucose using Reagent 1, which contains glucose oxidase and other chemicals. Similarly, Sample 2 can be assayed for lactate using Reagent 2, which consists of lactate oxidase and other chemicals. In this way, both glucose assay and lactate assay can be carried out concurrently. To demonstrate multiplexed assays, only cells and electrodes used for the bioassay have been fabricated.

In our example, the digital microfluidics-based biochip used for the multiplexed biochemical assay operations contains a $15 \times 15$ microfluidic array, as shown in Fig. 15 . This example is a full-packed array design compared to the prototype shown in Fig. 14. The fabricated prototype chip can be embedded in this array; the latter is expected to be the next-generation prototype for demonstrating more complex bioassays. Note that, unlike previous work, we do not manually assign the location of test droplet sources and sinks here. Instead, the proposed PMF algorithm can be used to determine the optimal location of the test hardware. The schedule of the set of bioassays, determined using the techniques in [16], is listed in Table
2; one procedure of the multiplexed assays takes $25.8 \mathrm{~s}$. The movement of droplets (including test droplets) is controlled using a $50 \mathrm{~V}$ actuation voltage with a switching frequency of $16 \mathrm{~Hz}$. The details of these colorimetric enzymatic reactions as well as the fabricated prototype can be found in [20].

We first apply the PMF algorithm described in Section 5 to obtain an off-line testing plan for the $15 \times 15$ microfluidic array. Its eulerized graph model for a single test droplet is shown in Fig. 16(a); next a test plan based on an Euler circuit is found using the PMF algorithm. The total testing time involves 448 time slots (i.e., 28 s), where the length of a time slot equals the droplet transportation time between two adjacent cells, i.e., $62.5 \mathrm{~ms}$. The test droplet sources and sinks can be located at any boundary cell other than dispensing ports for sample and reagent droplets. Next, we consider on-line testing for this example. The optimized concurrent test plan obtained using the PMF algorithm takes 480 time slots (i.e., $30 \mathrm{~s}$ ); compared to off-line testing, the test time is slightly higher due to the waiting time that is necessary to avoid conflicts with the normal bioassay. The optimal location for the test droplet source and sink is shown in Fig. 16(a). The test plan for the same biochip in [20] is only 18.7 s. Although the Euler circuit-based test plan
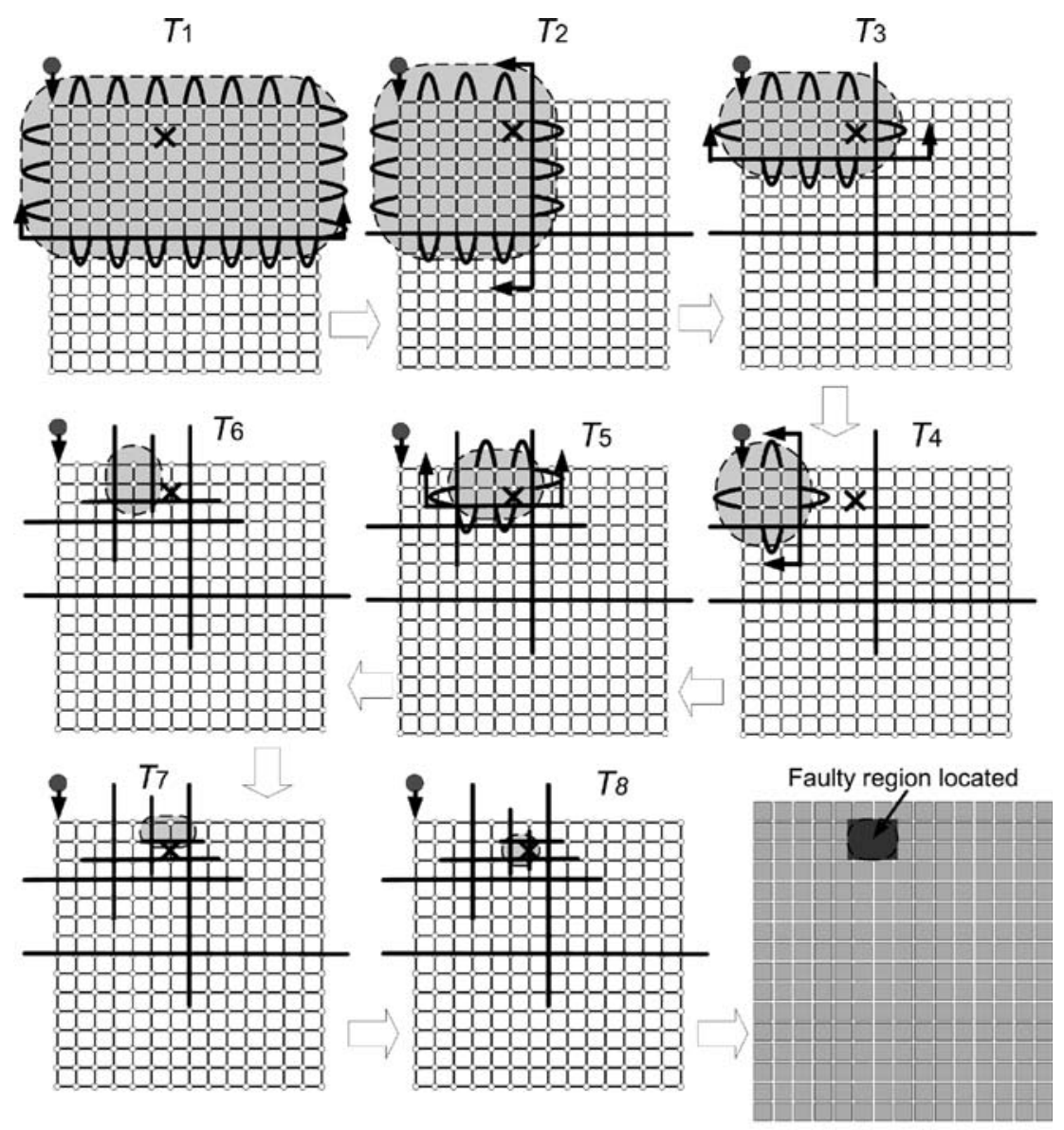

Test droplet source/sink

Fig. 17. Diagnosis procedure for a $15 \times 15$ microfluidic array. 
requires more testing time, it provides higher defect coverage, since it can detect defects such as electrode shorts that affect two adjacent cells. For safety-critical applications, defect coverage is more important than a slight increase in the test application time.

We further consider the application of multiple test droplets for this example. If we partition $15 \times 15$ microfluidic array into two $8 \times 15$ arrays as shown in Fig. 16(b), we can obtain an off-line test plan that allows two test droplets to traverse each partition while adhering to the constraints on droplet motion. The test application time for two test droplets is 238 time slots (i.e., 14.9 s), which is $47 \%$ less than that for a single test droplet. An optimized test plan for concurrent testing requires a total test time of 332 time slots, i.e., 20.8 s. Using the PMF algorithm, we find that the first partition requires 332 time slots for testing, while the second partition requires 308 time slots. The locations of two test droplet sources and sinks are also shown in Fig. 16(b).

Finally, we apply the proposed diagnosis technique to this example. Assume that the cell used as the first optical detection site is shorted to its adjacent cell. Thus the product droplet of the glucose assay cannot be transported to the appropriate location for optical detection, thus leading to a measurement error. The adaptive diagnosis scheme proposed in Section 5.3 can be applied to locate faulty regions, as shown in Fig. 17. There are in all $\left(\left\lceil\log _{2}(15-1)\right\rceil+\right.$ $\left.\left\lceil\log _{2}(15-1)\right\rceil\right)$, i.e., eight steps of adaptive testing procedures. Following the diagnosis procedure, we can reschedule the detection operation for the product of the glucose assay to another optical detector to avoid the error.

\section{Conclusions}

We have presented a defect-oriented testing and diagnosis methodology for digital microfluidics-based biochips. Experimental results have highlighted a major deficiency of prior work on the testing of microfluidic arrays; faults such as electrode shorts that affect two consecutive cells are not always detected by prior methods. To address this issue, we have formulated test planning in terms of the Euler circuit problem from graph theory. Both off-line and on-line testing methods have been presented. Diagnosis techniques to locate faulty cells in the microfluidic array have also been implemented using multi-step and adaptive Euler circuit-based testing procedures. The testing and diagnosis methods have been evaluated for a set of real-life bioassays. This work is expected to facilitate defect tolerance of digital microfluidics-based biochips, thereby increasing the reliability and system lifetime of these composite microsystems.

\section{Acknowledgements}

The authors thank Phil Paik of Duke University for help in carrying out the experiments involving electrode shorts.

\section{References}

1. T.H. Cormen, S. Clifford, C.E. Leiserson, and R.L. Rivest, Introduction to Algorithm, MIT, 2001.

2. N. Deb and R.D. Blanton, "Analysis of Failure Sources in Surfacemicromachined MEMS," Proc. IEEE Int. Test Conf., pp. 739-749, 2000.

3. N. Deb and R.D. Blanton, "Multi-modal Built-in Self-test for Symmetric Microsystems," Proc. IEEE VLSI Test Symp., pp. 139-147, 2004.

4. A. Dhayni, S. Mir, and L. Rufer, "MEMS Built-in-self-test using MLS," Proc. IEEE Eur. Test Symp., pp. 66-71, 2004.

5. International Technology Roadmap for Semiconductor (ITRS), http://public.itrs.net/Files/2003ITRS/Home2003.htm.

6. H.G. Kerkhoff, "Testing Philosophy Behind the Micro Analysis System," Proc. SPIE: Design, Test and Microfabrication of MEMS and MOEMS, vol. 3680, pp. 78-83, 1999.

7. H.G. Kerkhoff and H.P.A. Hendriks, "Fault Modeling and Fault Simulation in Mixed Micro-fluidic Microelectronic Systems," JETTA, vol. 17, pp. 427-437, 2001.

8. H.G. Kerkhoff and M. Acar, "Testable Design and Testing of Micro-electro-fluidic Arrays," Proc. IEEE VLSI Test Symp., pp. 403-409, 2003.

9. A. Kolpekwar and R.D. Blanton, "Development of a MEMS Testing Methodology," Proc. IEEE Int. Test Conf., pp. 923-93, 1997.

10. S. Mir, B. Charlot, and B. Courtois, "Extending Fault-based Testing to Microelectromechanical Systems," JETTA, vol. 16, pp. 279-288, 2000.

11. M.G. Pollack, "Electrowetting-Based Microactuation of Droplets for Digital Microfluidics," Ph.D. thesis, Duke University. 2001.

12. M. Pollack, A.D. Shenderov, and R.B. Fair, "Electrowetting-based Actuation of Droplets for Integrated Microfluidics," Lab Chip, vol. 2, pp. 96-101, 2002.

13. V. Srinivasan, V.K. Pamula, M.G. Pollack, and R.B. Fair, "A Digital Microfluidic Biosensor for Multianalyte Detection," Proc. IEEE MEMS Conference, pp. 327-330, 2003.

14. V. Srinivasan, V.K. Pamula, M.G. Pollack, and R.B. Fair, "Clinical Diagnostics on Human Whole Blood, Plasma, Serum, Urine, Saliva, Sweat, and Tears on a Digital Microfluidic Platform," Proc. Micro Total Analysis Systems, pp. 1287-1290, 2003.

15. V. Srinivasan et al., "An Integrated Digital Microfluidic Lab-on-achip for Clinical Diagnostics on Human Physiological Fluids," Lab Chip, pp. 310-315, 2004.

16. F. Su and K. Chakrabarty, "Architectural-level Synthesis of Digital Microfluidics-based Biochips," Proc. IEEE Int. Conf. on CAD, pp. 223-228, 2004.

17. F. Su and K. Chakrabarty, "Defect tolerance for Gracefullydegradable Microfluidics-based Biochips," accepted for publication in Proc. IEEE VLSI Test Symp., pp. 321-326, 2005.

18. F. Su, S. Ozev, and K. Chakrabarty, "Testing of Droplet-based Microelectrofluidic Systems," Proc. IEEE Int. Test Conf., pp. 11921200, 2003.

19. F. Su, S. Ozev, and K. Chakrabarty, "Test Planning and Test Resource Optimization for Droplet-based Microfluidic Systems," Proc. IEEE Eur. Test Sym., pp. 72-77, 2004.

20. F. Su, S. Ozev, and K. Chakrabarty, "Concurrent Testing of Droplet-based Microfluidic Systems for Multiplexed Biomedical Assays," Proc. IEEE Int. Test Conf., pp. 883-892, 2004.

21. F. Su, K. Chakrabarty, and V.K. Pamula, "Yield Enhancement of Digital Microfluidics-based Biochips Using Space Redundancy and Local Reconfiguration," accepted for publication in Proc. DATE Conference, pp. 1196-1201, 2005.

22. S.K. Tewksbury, "Challenges Facing Practical DFT for MEMS," Proc. Defect and Tolerance in VLSI Systems, pp. 11-17, 2001.

23. D.B. West, Introduction to Graph Theory, Prentice-Hall, New Jersey, 1996.

24. E. Verpoorte and N.F. De Rooij, "Microfluidics Meets MEMS," Proc. IEEE, vol. 91, pp. 930-953, 2003. 
Fei Su received his M.S. and Ph.D. degrees in electrical and computer engineering from Duke University in 2003 and 2006, respectively. He also received his B.S. degree in automation and M.S. degree in detection and Automation Devices from Tsinghua University in 1999 and 2001, respectively. He is currently a senior DFT (design-for-test) engineer of Intel Corporation (Folsom, CA, USA).

His research interest include CAD and testing for bio-SOC and mixed microsystems (e.g., microfluidic biochips), DFT and testing of high-speed IO and mixed-signals circuits. He has published over 20 papers in journals and refereed conference proceedings. Fei is also a co-author of a new book-Digital Microfluidic Biochip: Synthesis, Testing and Reconfiguration Techniques (CRC Press, Oct 2006). He is a member of IEEE TTTC (Test Technology Technical Council) and ACM SIGDA (Special Interest Group in Design Automation). He serves as an invited reviewer of IEEE Transactions on CAD, IEEE Designs \& Test of Computers, and IEEE North Atlantic Test Workshop.

William L. Hwang is a graduate student in chemical biology at the University of Oxford on a Rhodes Scholarship. He graduated first in his class from Duke University with degrees in Biomedical Engineering, Electrical and Computer Engineering, and Physics with a minor in Chemistry. He was a Goldwater Scholar and was named to the USA Today 2006 All-USA College Academic First Team. His other research interests include biosensing, biophysics, and applications of neuroscience research to education.

William plays outside hitter for the University of Oxford Men's Blues Volleyball Team and the Prosport National Volleyball League Oxford Men's Team. In 2003, William founded United InnoWorks Academy, Inc., a 501(c)(3) educational nonprofit organization and has served as CEO for 3 years. For his community work and leadership, he received numerous honors including the President's Volunteer Gold Service Award and the Algernon Sydney Sullivan Award. William was co-editor-in-chief of Eruditio, the Duke Undergraduate Humanities Journal, played outside hitter and setter for the Duke Men's Volleyball Team that won the 2004 NIRSA Intercollegiate Division IAA National Championship. He was a first violinist in the Duke Symphony Orchestra and served as vice president of the Tau Beta $\mathrm{Pi}$ National Engineering Society, NC Gamma Chapter.

Arindam Mukherjee completed his Ph.D. from the University of California at Santa Barbara in 2002 with a major in computer engineering. Since then he has been an assistant professor in the Department of Electrical and Computer Engineering at the University of North Carolina at Charlotte. His research interests include computer aided design, high performance FPGA-based reconfigurable computing systems, emulation and fault tolerance for supervisory control and data acquisition systems, and system design for labs-on-chip.

Krishnendu Chakrabarty received his B. Tech. degree from the Indian Institute of Technology, Kharagpur in 1990 and his M.S.E. and Ph.D. degrees from the University of Michigan, Ann Arbor, in 1992 and 1995, respectively, all in computer science and engineering. He is now professor of electrical and computer engineering at Duke University. Dr. Chakrabarty is a recipient of the National Science Foundation Early Faculty (CAREER) award and the Office of Naval Research Young Investigator award. His current research projects include: design and testing of system-on-chip integrated circuits, design automation of microfluidics-based biochips, microfluidics-based chip cooling and distributed sensor networks. Dr. Chakrabarty has authored four books- Microelectrofluidic Systems: Modeling and Simulation (CRC Press, 2002) and Test Resource Partitioning for System-ona-Chip (Kluwer, 2002), Scalable Infrastructure for Distributed Sensor Networks (Springer, 2005), Digital Microfluidics Biochips: Synthesis, Testing, and Reconfigutaion Techniques (CRC Press, October 2006) — and edited the book volumes SOC (System-ona-Chip) Testing for Plug and Play Test Automation (Kluwer, 2002) and Design Automation Methods and Tools for Microfluidics-Based Biochips (Springer, 2006). He has published over 230 papers in archival journals and refereed conference proceedings. He holds a US patent in built-in self-test and is a co-inventor of a pending US patent on sensor networks. He is a recipient of best paper awards at the 2005 IEEE International Conference on Computer Design and 2001 IEEE Design, Automation and Test in Europe (DATE) Conference. He is also a recipient of the Humboldt Research Fellowship, awarded by the Alexander von Humboldt Foundation, Germany.

Dr. Chakrabarty is a Distinguished Visitor of the IEEE Computer Society for 2006-2007 and a Distinguished Lecturer of the IEEE Circuits and Systems Society for 2006-2007. He is an associate editor of IEEE Transactions on Computer-Aided Design of Integrated Circuits and Systems, IEEE Transactions on VLSI Systems, IEEE Transactions on Circuits and System I, ACM Journal on Emerging Technologies in Computing Systems, and an editor of Journal of Electronic Testing: Theory and Applications (JETTA). He is a member of the editorial board for IEEE Design \& Test of Computers, Microelectronics Journal, Sensor Letters, and Journal of Embedded Computing, and he serves as a subject area editor for the International Journal of Distributed Sensor Networks. In the recent past, he has also served as an associate editor of IEEE Transactions on Circuits and Systems II: Analog and Digital Signal Processing. He is a senior member of IEEE, a member of ACM and ACM SIGDA, and a member of Sigma Xi. He serves as vice chair of technical activities in IEEE's Test Technology Technical Council and is a member of the program committees of several IEEE/ACM conferences and workshops. He is the chair of the emerging technologies subcommittee for the IEEE Int. Conf. $C A D$ (2005-2006), and subcommittee for new, emerging, and specialized technologies for the 2006-2007 IEEE/ACM Design Automation Conference. He served as tutorials chair for the 2005 IEEE International Conference on VLSI Design and program chair for the 2005 IEEE Asian Test Symposium. He is the program chair for the CAD, Design, and Test Conference for the 2007 IEEE Symposium on Design, Integration, Test, and Packaging of MEMS/ MOEMS (DTIP 07). He delivered keynote talks at the International Conference \& Exhibition on Micro Electro, Opto, Mechanical Systems and Components (Munich, Germany, October 2005), the International Conference on Design and Test of Integrated Systems (Tunis, Tunisia, September 2006), as well as invited talks on biochips CAD at several other conferences. 